\title{
Recent Results on Balanced Symmetric Boolean Functions
}

\author{
Yingming Guo, Guangpu Gao, Yaqun Zhao
}

\begin{abstract}
In this paper we prove all balanced symmetric Boolean functions of fixed algebraic degree are trivial when the number of variables grows large enough. We also present the nonexistence of trivial balanced elementary symmetric Boolean function except for $n=2^{t+1} l-1$ and $d=2^{t}$, where $t$ and $l$ are any nonnegative integers, which shows Cusick's conjecture for balanced elementary symmetric Boolean function is exactly the conjecture that all balanced elementary symmetric Boolean functions are trivial. In additional, we obtain a bound $n_{0}$, which depends only on the algebraic degree, such that Cusick's conjecture holds for any $n>n_{0}$.
\end{abstract}

\section{Index Terms}

Cryptography, Boolean functions, Balancedness, Symmetric, Elementary symmetric functions

\section{INTRODUCTION}

Boolean functions play an important role in the design of symmetric cryptographic systems. They are used for S-Box design in block cipher and utilized as nonlinear filters and combiners in stream ciphers. Symmetric Boolean functions, which have the property that their outputs only depend on the Hamming weight of their inputs, are an interesting subclass of Boolean functions for their advantage in both implementation complexity and storage space. In [1], Canteaut and Videau studied in detail symmetric Boolean functions. They established a link between the periodicity of simplified value vector of a symmetric function and its algebraic degree. Cai $e t$ al. computed a closed formula for the correlation between any two symmetric Boolean functions in terms of their periods [5]. Castro et $a l$. improved the formula for computing the exponential sums of symmetric Boolean functions [2](also see, Lemma 1).

Balancedness is a primary requirement to resist the attacks on each cryptosystem. In [8], Gathen and Rouche found all the balanced symmetric boolean functions up to 128 variables. Canteaut et al. proved that balanced symmetric functions of degree less than or equal to 7(excluding the trivial cases) only exist for eight variables [1]. Since the number of nontrivial balanced functions seems to be very small, they conjectured that balanced symmetric functions

This work is supported by National Program on Key Basic Research Project (973 Program No. 2012CB315905).

The authors are with the Department of Applied Mathematics, Zhengzhou Information Science and Technology Institute, P.O. Box 1001-741, Zhengzhou 450002, China (e-mail:guoyingming123@gmail.com). 
of fixed degree do not exist when the number of variables grows. For elementary symmetric Boolean functions, Cusick et al. proposed a conjecture in [3] about the nonexistence of nonlinear balanced elementary symmetric Boolean functions $\sigma_{n, d}$ except for $n=l \cdot 2^{t+1}-1$ and $d=2^{t}$, where $t$ and $l$ are any positive integers. They also obtained many results towards the conjecture in [4]. Later in [6] Gao et al. proved that when $n=3 \bmod 4$, the function is balanced if and only if $d=2^{k}, 1 \leq k \leq t$. It was proved in [4] that Cusick's conjecture [3] [4] holds for sufficient large number of variables, but a certain bound had not been obtained.

The paper is organized as follows. Some basics on Boolean functions are introduced in Section II. We discuss the asymptotic behavior of symmetric Boolean functions in Section III. We present the equivalence of Cusick's conjecture and some other results on balanced symmetric Boolean functions in Section IV. We end in Section V with a conclusion.

\section{Preliminaries}

Let $F_{2}^{n}$ be the vector space of $n$-tuples over the Field $F_{2}=\{1,0\}$ of two elements. We denote by $\oplus$ the sum over $F_{2}$. A Boolean function of $n$ variables is a mapping from $F_{2}^{n}$ into $F_{2}$. A Boolean function is said to be symmetric if its output is invariant under any permutation of its input bits. We denote by $B_{n}$ (resp. $S B_{n}$ ) the set of all Boolean functions (resp. symmetric Boolean functions) of $n$ variables. If $f: F_{2}^{n} \rightarrow F_{2}$, then $f$ can be uniquely represented as a multivariate polynomial over $F_{2}$, called algebraic normal form (ANF):

$$
f\left(x_{1}, \cdots, x_{n}\right)=\bigoplus_{\mu \in F_{2}^{n}} \lambda_{\mu}\left(\prod_{i=1}^{n} x_{i}^{\mu_{i}}\right), \quad \text { with } \quad \lambda_{\mu}=\bigoplus_{x \preceq \mu} f(x)
$$

Where $\left(x_{1}, \cdots, x_{n}\right) \preceq\left(\mu_{1}, \cdots, \mu_{n}\right)$ if and only if $\forall i, x_{i} \leq \mu_{i}$. The addition and multiplication operations are in $F_{2}$. The number of variables in the highest order product term with nonzero coefficient is called its algebraic degree (denoted by $\operatorname{deg}(f)$ ).

Definition 1: For integers $n$ and $d$, the elementary symmetric Boolean function with $n$ variables $\sigma_{n, d}$ is defined as the sum of all terms of degree $d$, that is

$$
\sigma_{n, d}=\bigoplus_{1 \leq i_{1} \leq \cdots \leq i_{d} \leq n} x_{i_{1}} \cdots x_{i_{d}}
$$

If $f(x)=\sigma_{n, d}$, then $v_{f}(i)=\left(\begin{array}{l}i \\ d\end{array}\right) \bmod 2$.

A Boolean function of $n$ variables is symmetric if and only if its algebraic normal form can be written as follows:

$$
\begin{aligned}
f\left(x_{1}, \cdots, x_{n}\right) & =\bigoplus_{i=0}^{n} \lambda_{f}(i)\left(\bigoplus_{\mu \in F_{2}^{n} w_{H}(\mu)=i} \prod_{j=1}^{n} x_{j}^{\mu_{j}}\right) \\
& =\bigoplus_{i=0}^{n} \lambda_{\mu} \sigma_{n, i},
\end{aligned}
$$

Where $\sigma_{n, i}$ is the elementary symmetric polynomial of degree $i$ in $n$ variables.

A Boolean function is said to be affine if its algebraic degree does not exceed 1 . The set of all $n$-variable affine functions is denoted by $A(n)$. The Hamming weight $w_{H}(x)$ of a binary vector $x \in F_{2}^{n}$ is the number of its nonzero coordinates, and the Hamming weight $w_{H}(f)$ of a Boolean function $f$ is the size of its support $\{x \in$ 
$\left.F_{2}^{n} \mid f(x)=1\right\}$. If $w_{H}(x)=2^{n-1}$, we call $f(x)$ balanced. The Hamming distance between two functions $f, g \in B_{n}$, denoted by $d(f, g)$ is defined as $d(f, g)=w t(f \oplus g)$. A symmetric function can be represented by a vector $v_{f}=\left(v_{f}(0), \cdots, v_{f}(n)\right)$, where $v_{f}(i)=f(x)$ for $x \in F_{2}^{n}$ with Hamming weight $w_{H}(x)=i$. It was proved that for any $f \in S B_{n}, v_{f}$ is periodic with period $2^{t}, 2^{t}<n$, if and only if $\operatorname{deg}(f) \leq 2^{t}-1 ; \operatorname{deg}(f)=2^{t}$ if and only if $v_{f}$ is periodic with period $2^{t+1}$ and is a part of $\left(v_{f}(0), \cdots, v_{f}\left(2^{t}-1\right), v_{f}(0) \oplus 1, \cdots, v_{f}\left(2^{t}-1\right) \oplus 1\right)^{\infty}$ [1].

Definition 2: For any $f \in B_{n}$, we denote by $\mathcal{F}(f)$ the following value related to the Fourier transform of $f$

$$
\mathcal{F}(f)=\sum_{x \in F_{2}^{n}}(-1)^{f(x)}=2^{n}-2 w_{H}(f) .
$$

Definition 3: [1] Let $n$ be an odd integer and $f \in S B_{n}$. We say that $f$ is a trivial balanced function if

$$
v_{f}(i)=v_{f}(n-i)+1,0 \leq i \leq n
$$

The even case corresponds to affine functions.

For an arbitrary positive integer $n$, we denote its 2-adic expression by $n=\sum_{i=0}^{l} n_{i} 2^{i}$. Let $a, b$ be positive integers, we denote $a \preceq b$ if for all $i(0 \leq k \leq l), a_{i} \leq b_{i}$ and otherwise $a \npreceq b$. The Lucas formula says [7, p. 79], $\left(\begin{array}{l}n \\ k\end{array}\right)=\left(\begin{array}{l}n_{0} \\ k_{0}\end{array}\right)\left(\begin{array}{l}n_{1} \\ k_{1}\end{array}\right) \cdots\left(\begin{array}{l}n_{l} \\ k_{l}\end{array}\right) \bmod 2$. Let $f(x)=\sigma_{n, d}$, by Lucas formula, $v_{f}(i)=1$ if and only if $d \preceq i$.

It was proved that if $\sigma_{n, d}$ is balanced, then $d \leq\left\lceil\frac{n}{2}\right\rceil$ [3].

\section{Asymptotic Behavior of Symmetric BoOleAn FunCtions}

In this section, we characterize the behavior of symmetric Boolean functions with large number of variables. As a consequence of our discussion using the technique for the correlation of symmetric functions in [5], we prove the following conjecture.

Conjecture 1: [1, VIII] Excluding the trivial cases, balanced symmetric functions of fixed degree do not exist when the number of variables grows.

To prove Conjecture 1, we introduce the following lemma.

Lemma 1: [2] Let $f(x)=\sigma_{n, k_{s}}+\cdots+\sigma_{n, k_{1}}, 1 \leq k_{1} \leq \cdots \leq k_{s}$ and let $r=\left\lfloor\log _{2} k_{s}\right\rfloor+1 . \mathcal{F}(f)$ is given by

$$
\mathcal{F}(f)=\sum_{i=0}^{n}(-1)^{v_{f}(i)}\left(\begin{array}{l}
n \\
i
\end{array}\right)=\frac{1}{2^{r}} \sum_{j=0}^{2^{r}-1} s_{j} \lambda_{j}^{n},
$$

where $\xi_{j}=\exp \left(\frac{\pi \sqrt{-1} j}{2^{r-1}}\right), \lambda_{j}=1+\xi_{j}^{-1}$, and $d^{1}$

$$
s_{j}=\sum_{i=0}^{2^{r}-1}(-1)^{v_{f}(i)} \xi_{j}^{i}
$$

\footnotetext{
${ }^{1}$ Here we correct typos of the paper [2], where $\lambda_{j}=1+\xi_{j}$ should be $\lambda_{j}=1+\xi_{j}^{-1}$ and $s_{j}=\sum_{i=0}^{2^{r}-1}(-1)^{v_{f}(i)} \xi_{j}^{-i}$ should be $s_{j}=\sum_{i=0}^{2^{r}-1}(-1)^{v_{f}(i)} \xi_{j}^{i}$.
} 
If $f$ is not affine, then $r \geq 2$. Note that $\xi_{2^{r}-j}=\overline{\xi_{j}}, s_{2^{r}-j}=\overline{s_{j}}, \lambda_{2^{r}-j}=\overline{\lambda_{j}}$ and $\lambda_{2^{r-1}}=0$. We have the following observation on Lemma 1:

$$
\begin{aligned}
2^{r-1} \mathcal{F}(f) & =\frac{1}{2} \sum_{j=0}^{2^{r}-1} s_{j} \lambda_{j}^{n} \\
& =\frac{1}{2} s_{0} \lambda_{0}^{n}+\sum_{j=1}^{2^{r-1}-1} \operatorname{Re}\left(s_{j} \lambda_{j}^{n}\right)
\end{aligned}
$$

where the second equality holds because the second half of the $j$-sum $\left(2^{r-1} \leq j \leq 2^{r}-1\right)$ is the complex conjugate of the first half. Let us define

$$
t_{j}(n)=\frac{1}{2^{r-1}} \operatorname{Re}\left(s_{j} \lambda_{j}^{n}\right), 0 \leq j \leq 2^{r-1}-1
$$

Thus

$$
\mathcal{F}(f)=\frac{1}{2} t_{0}(n)+\sum_{j=1}^{2^{r-1}-1} t_{j}(n) .
$$

If $\mathcal{F}(f)=0$, there are potentially two reasons for this: either all the $t_{j}(n)$ are zero or several nonzero $t_{j}(n)$ $\left(\frac{1}{2} t_{j}(n)\right.$ for $\left.j=0\right)$ can cancel each other. The next lemma states that the latter cannot happen for large enough $n$. However, we should point out that the following lemma, although having a different $t_{j}(n)$, contributes to Cai $e t$ al. [5].

Lemma 2: Let $f \in S B_{n}$, and $f$ is not affine. There exists an integer $n_{0}$ such that for any $n>n_{0}$,

$$
\mathcal{F}(f)=0 \Leftrightarrow t_{j}(n)=0,0 \leq j \leq 2^{r-1}-1 .
$$

Proof: Suppose $\mathcal{F}(f)=0$. We can express $t_{j}(n)$ as ${ }^{2}$

$$
t_{j}(n)=\frac{1}{2^{r-1}}\left|s_{j}\right|\left|2 \cos \left(\frac{\pi j}{2^{r}}\right)\right|^{n} \cos \left(\arg \left(s_{j}\right)-\frac{\pi n j}{2^{r}}\right) .
$$

Clearly, we have

$$
t_{j}(n) \leq \frac{1}{2^{r-1}}\left|s_{j}\right|\left|2 \cos \left(\frac{\pi j}{2^{r}}\right)\right|^{n} .
$$

On the other hand, if $t_{j}(n) \neq 0$, since the cosine is periodic in $n$, for any $j$ there exists a constant $c_{j}>0\left(c_{j}\right.$ does not depend on $n$ ) such that

$$
t_{j}(n) \geq c_{j}\left|2 \cos \left(\frac{\pi j}{2^{r}}\right)\right|^{n} .
$$

Hence, each $\left|t_{j}(n)\right|$ is either zero or in a constant range of $\left|2 \cos \left(\frac{\pi j}{2^{r}}\right)\right|^{n}$.

Since when $n$ is large enough, $\left|2 \cos \left(\frac{\pi j}{2^{r}}\right)\right|^{n}$ dominates $\left|2 \cos \left(\frac{\pi(j+1)}{2^{r}}\right)\right|^{n}$ for any $j<2^{r}-1$, any $t_{j}(n) \neq 0$ dominates all the $t_{j^{\prime}}(n)$ for $j<j^{\prime}<2^{r-1}$. Thus if $j_{0}$ is the least $j$ such that $t_{j}(n) \neq 0$, then the subsequent terms cannot cancel $t_{j_{0}}(n) \quad\left(\frac{1}{2} t_{j_{0}}(n)\right.$ for $\left.j_{0}=0\right)$, and hence, $\mathcal{F}(f) \neq 0$. Therefore, $t_{j}(n)=0$, for all $0 \leq j \leq 2^{r-1}-1$.

\footnotetext{
${ }^{2}$ Any complex number $z \neq 0$ can uniquely be written as $z=|z|(\cos \varphi+i \sin \varphi)=|z| e^{i \varphi}$. where $0 \leq \varphi \leq 2 \pi$. $\varphi$ is called the argument of $z, \varphi=\arg z$. Hence $\operatorname{Re}(z)=|z| \cos \arg (z)$
} 
If $s_{j} \neq 0$, then for any $j, 0 \leq j \leq 2^{r-1}-1$, we have

$$
\begin{aligned}
& t_{j}(n)=0 \\
& \Leftrightarrow \cos \left(\arg \left(s_{j}\right)-\frac{\pi n j}{2^{r}}\right)=0 \\
& \quad \Leftrightarrow \quad \exists l \quad \arg \left(s_{j}\right)-\frac{\pi n j}{2^{r}}=\frac{\pi}{2}+l \pi \\
& \quad \Leftrightarrow \quad \exists l \quad \exp \left(2 i \arg \left(s_{j}\right)\right)=\exp \left(2 i\left(\frac{\pi n j}{2^{r}}+\frac{\pi}{2}+l \pi\right)\right) \\
& \quad \Leftrightarrow\left|s_{j}\right| \exp \left(i \arg \left(s_{j}\right)\right)=-\left|s_{j}\right| \exp \left(-i \arg \left(s_{j}\right)\right) \exp \left(\frac{\pi i}{2^{r}} n j\right) \\
& \quad \Leftrightarrow s_{j}=-\xi_{j}^{n} \overline{s_{j}}
\end{aligned}
$$

Note that if $s_{j}=0$, we get $t_{j}(n)=0, s_{j}=-\xi_{j}^{n} \overline{s_{j}}=0$. Thus

$$
t_{j}(n)=0 \Leftrightarrow s_{j}=-\xi_{j}^{n} \overline{s_{j}}, \quad 0 \leq j \leq 2^{r-1}-1
$$

holds no matter whether $s_{j}$ is zero.

Next, we have the following lemma.

Lemma 3: Let $f \in S B_{n}$ and $f$ is not affine. The following properties are equivalent.

(i) $s_{j}=-\xi_{j}^{n} \overline{s_{j}}, \quad 0 \leq j \leq 2^{r}-1$,

(ii) $v_{f}(i)=1 \oplus v_{f}(n-i), \quad 0 \leq i \leq 2^{r}-1$.

Proof: If property (ii) is true, then it is clear that

$$
s_{j}=\sum_{i=0}^{2^{r}-1}(-1)^{v_{f}(n-i)+1} \xi_{j}^{i},
$$

Since $(-1)^{v_{f}(i)} \xi_{j}^{i}$ has period $2^{r}, 0 \leq j \leq 2^{r}-1$, thus

$$
\begin{aligned}
-\xi_{j}^{n \overline{s_{j}}} & =-\xi_{j}^{n} \sum_{i=0}^{2^{r}-1}(-1)^{v_{f}(i)} \xi_{j}^{-i} \\
& =-\xi_{j}^{n} \sum_{i=n}^{n+2^{r}-1}(-1)^{v_{f}(i)} \xi_{j}^{-i} \\
& =-\sum_{i=n}^{n+2^{r}-1}(-1)^{v_{f}(i)} \xi_{j}^{n-i} \\
& =\sum_{i=0}^{2^{r}-1}(-1)^{v_{f}(n-i)+1} \xi_{j}^{i}=s_{j}
\end{aligned}
$$

If property (i) is true, then for any $0 \leq j \leq 2^{r}-1$,

$$
\sum_{i=0}^{2^{r}-1}(-1)^{v_{f}(i)} \xi_{j}^{i}=\sum_{i=0}^{2^{r}-1}(-1)^{v_{f}(n-i)+1} \xi_{j}^{i}
$$

Note that these sums are the Fourier transforms of the functions $f(i)$ and $f(n-i)$, respectively. We can perform an inverse Fourier transform by using the relation

$$
\sum_{j=0, j \neq 2^{r-1}}^{2^{r}-1} \xi_{j}^{i-i^{\prime}}=\left\{\begin{array}{rl}
2^{r}-1 & i=i^{\prime} \\
(-1)^{i-i^{\prime}+1}, & i \neq i^{\prime}
\end{array}\right.
$$


Now multiply the left and right hand sides of equation (10) by $\xi_{j}^{-i^{\prime}}$ and sum over $j$ from 0 to $2^{r}-1$. Then we have

$$
2^{r}(-1)^{v_{f}\left(i^{\prime}\right)}-\sum_{i=0}^{2^{r}-1}(-1)^{v_{f}(i)}=2^{r}(-1)^{v_{f}\left(n-i^{\prime}\right)+1}-\sum_{i=0}^{2^{r}-1}(-1)^{v_{f}(n-i)+1}
$$

Since $\sum_{i=0}^{2^{r}-1}(-1)^{v_{f}(n-i)+1}=-\sum_{i=0}^{2^{r}-1}(-1)^{v_{f}(i)}$, thus

$$
(-1)^{v_{f}\left(i^{\prime}\right)}+(-1)^{v_{f}\left(n-i^{\prime}\right)}=\frac{1}{2^{r-1}} \sum_{i=0}^{2^{r}-1}(-1)^{v_{f}(i)}
$$

The left hand side can be \pm 2 or 0 . If it is \pm 2 , then $f$ is constant, which contradicts to the hypothesis. Hence, the left hand side is 0 and we conclude $(-1)^{v_{f}\left(i^{\prime}\right)}=(-1)^{1+v_{f}\left(n-i^{\prime}\right)}$. The property (ii) follows.

Now we prove Conjecture1.

Theorem 1: For large enough $n$, balanced symmetric functions of fixed degree are trivial.

Proof: The case that $f \in S B_{n}$ being affine is clear, we now assume that $f$ is a non-affine function.

Let $f$ be a non-affine function with period $2^{r}$. Following Lemma 1 to Lemma 3, there exists an integer $n_{0}$ such that for any $n>n_{0}$,

$$
\mathcal{F}(f)=0 \Leftrightarrow v_{f}(i)=1 \oplus v_{f}(n-i), \quad i=0, \cdots, 2^{r}-1 .
$$

Note that when $n$ is even, we get $v_{f}\left(\frac{n}{2}\right)=1+v_{f}\left(\frac{n}{2}\right)$, which is a contradiction. That is, for sufficient large $n$, non-affine balanced symmetric functions are trivial for odd $n$ and do not exist for even $n$. Therefore, Theorem 1 is proved.

\section{The EQuivalence of Cusick’s CONJeCture}

In this section, we show the conjecture for elementary symmetric Boolean functions is equal to the conjecture that all balanced elementary symmetric Boolean functions are trivial balanced. Associating with the theorem in last section, we present the conjecture is validated with sufficient large number of variables.

Conjecture 2: [3] There are no nonlinear balanced elementary symmetric Boolean functions except for $\sigma_{l \cdot 2^{t+1}-1,2^{t}}$, where $t$ and $l$ are any positive integers.

As mentioned in the proof of Theorem 3 in [3], $f(x)=\sigma_{l \cdot 2^{t+1}-1,2^{t}}(x)$ is trivial balanced. In the following theorem we prove that all trivial balanced elementary symmetric Boolean functions are of the form $\sigma_{l \cdot 2^{t+1}-1,2^{t}}$.

Theorem 2: There are no trivial balanced elementary symmetric Boolean functions except for $\sigma_{l \cdot 2^{t+1}-1,2^{t}}$, where $t$ and $l$ are any nonnegative integers.

Proof: Supposed $f(x)=\sigma_{n, d}(x)$ is trivial balanced. If $d=1$, the conclusion follows regardless of the parity of $n$. Let $d>1$, than $n$ must be odd, and $v_{f}(i)=v_{f}(n-i), 0 \leq i \leq n$. Since $v_{f}(i)=\left(\begin{array}{l}i \\ d\end{array}\right) \bmod 2$ and $v_{f}(i)=1$ if and only if $d \preceq i$, we get either $d \preceq i$ or $d \preceq(n-i)$.

Let the 2-adic expressions of $d, n$ be $d=\sum_{i=0}^{t} d_{i} 2^{i}, n=\sum_{i=0}^{k} n_{i} 2^{i}, k>t$ and $\bar{d}=d_{t} \cdots d_{0}, \bar{n}=n_{k} \cdots n_{0}$. We argue that $d=2^{t}$. Otherwise, suppose $d_{t}, d_{j}$ are ones(i.e. $\left.\bar{d}=1_{t} \cdots 1_{j} \cdots\right)$. On the one hand, let $\bar{i}=$ 
$0_{t} \cdots 1_{j} \underbrace{0_{j-1} \cdots 0_{0}}_{0}$. Since $d \npreceq i$, we get $d \preceq(n-i)$, which implies $n_{j}=0$. On the other hand, let $\overline{i^{\prime}}=$ $1_{t} \cdots \underbrace{0_{j} \cdots 0_{0}}_{0}$. Since $d \npreceq i^{\prime}$,we get $d \preceq\left(n-i^{\prime}\right)$, which implies $n_{j}=1$. It is a contradiction.

Furthermore, we claim that $n=l \cdot 2^{t+1}-1$ (i.e. $\bar{n}=n_{k} \cdots n_{t+1} \underbrace{1_{t} \cdots 1_{0}}_{1}$ ). Otherwise, suppose $n_{j}$ is the first zero from the $t$-th bit to the last bit(i.e. $\bar{n}=n_{k} \cdots n_{t+1} \underbrace{1_{t} \cdots 1_{j+1}}_{1} 0_{j} n_{j-1} \cdots n_{0})$. Let $\bar{i}=0_{t} \underbrace{1_{t-1} \cdots 1_{j}}_{1} \underbrace{0_{j-1} \cdots 0_{0}}_{0}$, then we get $\overline{n-i}=n_{k} \cdots n_{t+1} 0_{t} \underbrace{1_{t-1} \cdots 1_{j+1} 1_{j}}_{1} n_{j-1} \cdots n_{0}$, so both $d \preceq i$ and $d \preceq n-i$ are false, which is a contradiction.

Hence, we conclude that all trivial balanced elementary symmetric Boolean functions are of the form $\sigma_{l \cdot 2^{t+1}-1,2^{t}}$.

Remark 1: By Theorem 2, Conjure 2 shows in essence that all balanced elementary symmetric Boolean functions are trivial balanced. According to Theorem 1 and Theorem 2, we conclude that there exists an integer $n_{0}$ such that Conjure 2 holds for any $n>n_{0}$.

At the end of the section, we give an estimation for $n_{0}$. Let $f(x)=\sigma_{l \cdot 2^{t+1}-1,2^{t}}(x)$. By the equation (2) in [1], we get

$$
\begin{aligned}
\mathcal{F}(f) & \\
& =\sum_{i=0}^{2^{r}-1}(-1)^{v_{f}(i)}\left(2^{n-r}+2^{1-r} \sum_{j=1}^{2^{r-1}-1}\left(2 c_{j}\right)^{n} c_{j}^{\prime}\right) \\
& =2^{n-r}\left(\sum_{i=0}^{2^{r}-1}(-1)^{v_{f}(i)}+2 \sum_{j=1}^{2^{r-1}-1} \sum_{i=0}^{2^{r}-1}(-1)^{v_{f}(i)} c_{j}^{n} c_{j}^{\prime}\right)
\end{aligned}
$$

where $c_{j}=\cos \left(j \frac{\pi}{2^{r}}\right), c_{j}^{\prime}=\cos \left(j(n-2 i) \frac{\pi}{2^{r}}\right)$. It is exactly the equation (3), where for $0 \leq j \leq 2^{r-1}-1, A_{j}=$ $\frac{j \pi}{2^{r}}(n-2 i)$,

$$
t_{j}(n)=2^{n-r+1} \cos ^{n}\left(j \frac{\pi}{2^{r}}\right) \sum_{i=0}^{2^{r}-1}(-1)^{\left(\begin{array}{l}
i \\
d
\end{array}\right)} \cos A_{j} .
$$

If $t_{0}(n) \geq 2^{j+1}\left|t_{j}(n)\right|$, it is obvious that $\mathcal{F}(f)=0$ if and only if $t_{0}(n)=t_{j}(n)=0,1 \leq j \leq 2^{r-1}-1$. Thus we have the following result.

Theorem 3: Let $r=\left\lfloor\log _{2} d\right\rfloor+1$. All these nonlinear balanced elementary symmetric Boolean functions are of the form $\sigma_{l \cdot 2^{t+1}-1,2^{t}}$, where $t$ and $l$ are any positive integers for any $n>-2\left(\log _{2} \cos \left(\frac{\pi}{2^{r}}\right)\right)^{-1}$.

Proof: Consider $t_{j}(n), 1 \leq j \leq 2^{r-1}-1$,

(1) If $\sum_{i=0}^{2^{r}-1}(-1)^{\left(\begin{array}{l}i \\ d\end{array}\right)} \cos A_{j} \geq 0$, then

$$
\begin{gathered}
\sum_{i=0}^{2^{r}-1}(-1)^{\left(\begin{array}{l}
i \\
d
\end{array}\right)}-\sum_{i=0}^{2^{r}-1}(-1)^{\left(\begin{array}{l}
i \\
d
\end{array}\right)} \cos A_{j} \\
=2 \sum_{i=0}^{2^{r}-1}(-1)^{\left(\begin{array}{l}
i \\
d
\end{array}\right) \sin ^{2}\left(\frac{A_{j}}{2}\right)}
\end{gathered}
$$




$$
\begin{aligned}
& \geq 2 \sum_{i=0}^{2^{r-1}-1}(-1)^{\left(\begin{array}{l}
i \\
d
\end{array}\right)}\left(\sin ^{2}\left(\frac{A_{j}}{2}\right)+\cos ^{2}\left(\frac{A_{j}}{2}\right)\right) \\
& =0
\end{aligned}
$$

(2) If $\sum_{i=0}^{2^{r}-1}(-1)^{\left(\begin{array}{l}i \\ d\end{array}\right)} \cos A_{j}<0$, then

$$
\begin{aligned}
& \sum_{i=0}^{2^{r}-1}(-1)^{\left(\begin{array}{l}
i \\
d
\end{array}\right)}+\sum_{i=0}^{2^{r}-1}(-1)^{\left(\begin{array}{l}
i \\
d
\end{array}\right)} \cos \left(\frac{A_{j}}{2}\right) \\
& =2 \sum_{i=0}^{2^{r}-1}(-1)^{\left(\begin{array}{l}
i \\
d
\end{array}\right)} \cos ^{2}\left(\frac{A_{j}}{2}\right) \\
& \geq 2 \sum_{i=0}^{2^{r-1}-1}(-1)^{\left(\begin{array}{l}
i \\
d
\end{array}\right)}\left(\cos ^{2}\left(\frac{A_{j}}{2}\right)+\sin ^{2}\left(\frac{A_{j}}{2}\right)\right) \\
& =0
\end{aligned}
$$

These two equalities hold if and only if $d=2^{r-1}$. Therefore $t_{0}(n) \geq\left|t_{j}(n)\right|$ for $1 \leq j \leq 2^{r-1}-1$.

When $n>-2 /\left(\log _{2} \cos \left(\frac{\pi}{2^{r}}\right)\right)$, we have $\cos ^{n}\left(\frac{\pi}{2^{r}}\right)<\frac{1}{4}$, and hence $t_{0}(n)>\left|4 t_{1}(n)\right|$. In additional, since for any $j, 1 \leq j \leq 2^{r-1}-1$,

$$
\begin{aligned}
\frac{\cos \left(\frac{j}{2^{r}} \pi\right)}{\cos \left(\frac{j+1}{2^{r}} \pi\right)} & =\frac{\cos \left(\frac{j}{2^{r}} \pi\right)}{\cos \left(\frac{\pi}{2^{r}}\right) \cos \left(\frac{j}{2^{r}} \pi\right)-\sin \left(\frac{\pi}{2^{r}}\right) \sin \left(\frac{j}{2^{r}} \pi\right)} \\
& >\frac{1}{\cos \left(\frac{\pi}{2^{r}}\right)}>4,
\end{aligned}
$$

$t_{0}(n)>2^{j+1}\left|t_{j}(n)\right|$ holds for any $j, 1 \leq j \leq 2^{r-1}-1$.

Hence $\mathcal{F}(f)=0$ if and only if $t_{0}(n)=t_{j}(n)=0$. From the discussion in Section III, if $f$ is balanced, then $f$ is trivial. Therefore, Conjecture 2 is validated for all $n>-2\left(\log _{2} \cos \left(\frac{\pi}{2^{r}}\right)\right)^{-1}$, where $2^{r-1} \leq d<2^{r}$.

\section{CONCLUSion}

In this paper, we study the balancedness of symmetric Boolean functions. We prove the conjecture that all balanced symmetric Boolean functions of fixed degree are trivial when the number $n$ of variables is sufficient large. We also present the form of trivial balanced elementary symmetric Boolean functions. In addition, we estimate the lower bound of $n$ with which Cusick's conjecture for elementary symmetric Boolean functions is validated. But the bound is rough. We show some properties of the balancedness of a symmetric Boolean function in the view of the distance from a balanced one, which can be a new and clear way to proof some former results. Although an equivalence of Cusick's conjecture is established, it is till an open problem. It would be helpful to remove the dependence on the degree from the bound, since then the complete proof of the conjecture would be reduced to a finite computation.

\section{ACKNOWLEDGMENT}

The authors would like to thank Prof. T.W. Cusick for a careful reading of the manuscript and for all his helpful suggestions. 


\section{REFERENCES}

[1] A. Canteaut and M. Videau, "Symmetric Boolean functions," IEEE Trans. Inf. Theory, vol.51, no.8, pp.2791-2881, 2005.

[2] F.N. Castro, and L.A. Medina, "Linear recurrences and asymptotic behavior of exponential sums of symmetric boolean functions," The Electronic Journal of Combinatorics, vol.18, no.2, pp.P8, 2011.

[3] T.W. Cusick, Y. Li, and P. Stanica, "Balanced symmetric Boolean functions over GF(p)," IEEE Trans. Inf. Theory, vol.3, no.54, pp.13041307, 2008.

[4] T.W. Cusick, Y. Li, and P. Stanica, "On a conjecture for balanced symmetric Boolean functions," J. Math. Crypt., vol. 3. no. 4, pp. 273-290, 2009.

[5] J. Y. Cai, F.Green. and T. Thierauf., "On the correlation of symmetric functions," Theory of Computing Systems, vol.29, pp.245-258, 1996.

[6] G. Gao, W. Liu, and X. Zhang, "The degree of balanced elementary symmetric Boolean functions of 4k + 3 Variables," IEEE Trans. Inf. Theory, vol.57, no.7, pp. 4822-4825, 2011

[7] L. Comtet, Advanced Combinatorics, Amsterdam. The Netherlands: Reidel, 1974.

[8] J. von zur Gathen and J. Roche, "Polynomials with two values," Combinatorica, vol.17, pp.345-362, 1997.

[9] A. Braeken, and B. Preneel, "On the Algebraic Immunity of Symmetric Boolean Functions," in Proc. INDOCRYPT 2005 (Lecture Notes in Computer Science), Berlin, Germany, 2005, vol.3797, pp. 35-48. [Online]. Available: http://eprint.iacr.org/2005/245.pdf

[10] M. Liu, D. Lin and D. Pei, "Fast Algebraic Attacks and Decomposition of symmetric boolean functions," IEEE Trans. Inf. Theory, vol.57 no.7, pp. 4817-4821, 2011. 